Hydraulic Engineering Repository

Ein Service der Bundesanstalt für Wasserbau

Annandale, George W. Quantification of the Erosive Capacity of Water

Verfügbar unter / Available at:

https://hdl.handle.net/20.500.11970/99991

Vorgeschlagene Zitierweise / Suggested citation:

Annandale, George W. (2006): Quantification of the Erosive Capacity of Water. In: Verheij, H.J.; Hoffmans, Gijs J. (Hg.): Proceedings 3rd International Conference on Scour and Erosion (ICSE-3). November 1-3, 2006, Amsterdam, The Netherlands. Gouda (NL): CURNET. S. 18-22. 


\title{
Quantification of the Erosive Capacity of Water
}

\author{
George W. Annandale* \\ * Engineering and Hydrosystems Inc., Denver, Colorado, USA
}

\begin{abstract}
When expressing average flow velocity, wall shear stress and available stream power as a function of hydraulic roughness it is found that flow velocity decreases, wall shear stress increases and stream power remains constant [2]. These three parameters are often used as indicators of the relative magnitude of the erosive capacity of water, and the differing trends provide practicing engineers with a practical problem. The trend characteristics imply that it is unknown whether the erosive capacity of water will increase, decrease or remain constant when using conventional parameters for quantifying the relative magnitude of the erosive capacity of flowing water subject to varying hydraulic roughness.
\end{abstract}

This paper addresses the apparent dichotomy by using boundary layer theory to quantify turbulent boundary shear stress and applied boundary stream power as a function of hydraulic roughness. By making use of findings by [4] and the conclusions from the boundary layer analysis [1], it is concluded that either turbulent boundary shear stress or applied boundary stream power can be used to quantify the relative magnitude of the erosive capacity of water. Using applied boundary stream power is preferred because it resembles turbulence intensity and therefore quantifies the relative magnitude of pressure fluctuations in turbulent flow, which is the principal agent leading to incipient motion [1]. It is shown that average flow velocity is an undesirable indicator of the relative magnitude of the erosive capacity of water because it provides contradictory results.

\section{INTRODUCTION}

The term "erosive capacity of water" is an elusive concept. From empirical experience it is known that the potential for incipient motion of earth materials, and thus the potential for scour, increases as the "flow intensity" of water increases. The concept of increased flow intensity can take on different meanings, dependent on the observer. Some observers prefer to express it in terms of average flow velocity, while others prefer to express it as shear stress or stream power. It has been found that use of these three indicator parameters to quantify the relative magnitude of the erosive capacity of flowing water results in inconsistent conclusions [1].

The paper illustrates that this inconsistency can be resolved by quantifying the relative magnitude of the erosive capacity of turbulent flowing water when taking full account of flow processes in the near-boundary region.

\section{FLOW CONDITIONS AT INCIPIENT MOTION}

Reference [4] has shown that incipient motion under laminar flow conditions is a function of wall shear stress, while the same under turbulent flow conditions depends purely on fluctuating turbulent pressures when flow conditions in the near boundary region is characterized as smooth turbulent flow. The analysis by [4] also demonstrates that turbulent fluctuating pressures combined with a transverse force of water along the bed results in concurrent lifting and rotation of particles at the point of incipient motion when flow conditions in the near boundary region changes to rough turbulent flow. The validity of these proposed mechanisms were illustrated by making use of basic principles of hydraulics and the Shields diagram.

Reference [3] showed that fluctuating pressures in turbulent flow play the dominant role in scour of rock, regardless of whether it scours by means of dynamic impulsion (block removal), brittle fracture or fatigue failure. Methods for quantifying the magnitude of fluctuating pressures resulting from turbulent plunging jets are available (see e.g. [1] and [3]).

Methods for quantifying the magnitude of turbulent fluctuating pressures for most other flow conditions, such as flow around bridge piers, past bridge abutments, around channel bends, underneath hydraulic jumps, etc. are not generally known. Practicing engineers therefore prefer to use indicator parameters to quantify the relative magnitude of such pressure fluctuations, and thus the magnitude of the erosive capacity of water in turbulent flow.

\section{INDICATOR PARAMETERS}

The objective of using indicator parameters in scour technology is to quantify the relative magnitude of the erosive capacity of water. Typical indicator parameters that are generally used in engineering practice include average flow velocity, wall shear stress and available stream power.

Average flow velocity $\bar{u}$ can be expressed as,

$$
\bar{u}=18 \log \left(\frac{12 \cdot R}{k_{s}}\right) \cdot \sqrt{R \cdot s_{f}}
$$




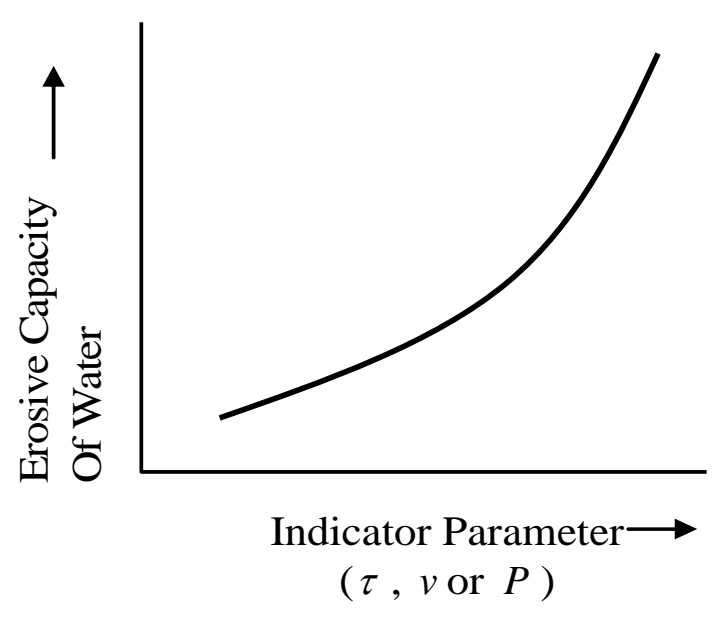

Figure 1. Desired relationship between indicator parameters and the actual magnitude of the erosive capacity of water.
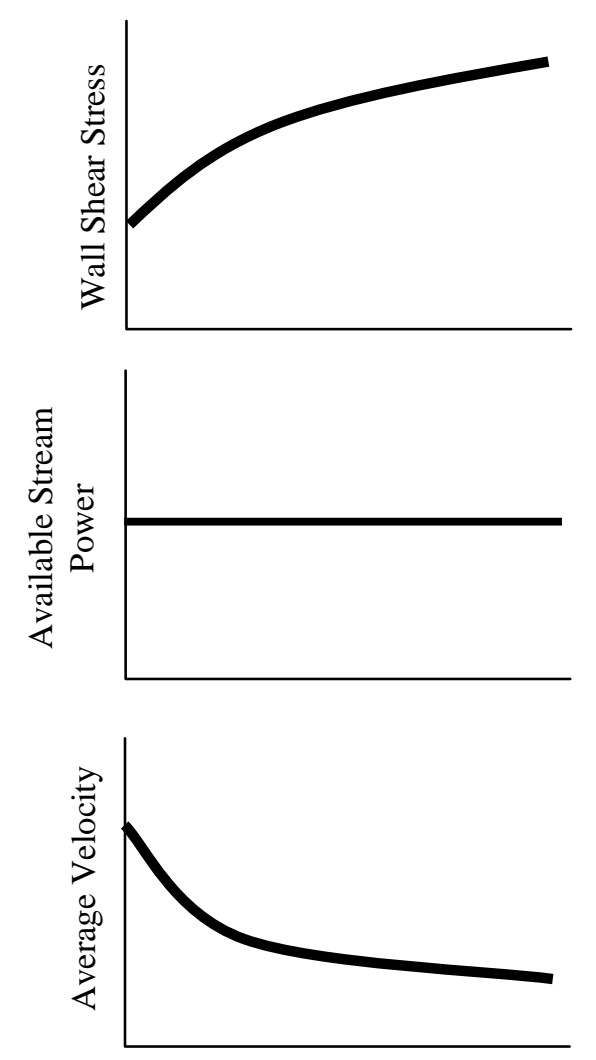

Absolute Roughness $k_{\mathrm{s}}$

Figure 2. Actual behavior of commonly used indicator parameters for quantifying the relative magnitude of the erosive capacity of water, expressed as a function of absolute roughness.

Where $R=$ hydraulic radius; $s_{f}=$ energy slope; $k_{s}=$ absolute roughness.

Wall shear stress $\bar{\tau}_{w}$ is calculated as,

$$
\bar{\tau}_{w}=\rho \cdot g \cdot R \cdot s_{f}
$$

Where $\rho=$ mass density of water; $g=$ acceleration due to gravity.

$$
\text { Available stream power } P \text { is expressed as, }
$$

$$
P=\tau \cdot u
$$

Due to the fact that indicator parameters do not quantify the exact magnitude of the erosive capacity of water, but only its relative magnitude, these parameters should be characterized by internal consistency. The behavior of indicator parameters is internally consistent if the relative change in parameter value resembles the actual change in the erosive capacity of flowing water. This means that the magnitude of an indicator parameter must consistently increase when the actual magnitude of the erosive capacity of the water increases. Similarly, the values of the indicator parameters must consistently decrease when the actual magnitude of the erosive capacity of water decreases (Fig. 1).

References [1] and [2] show that the conventional indicator parameters used in engineering (i.e. average flow velocity, wall shear stress and available stream power) are not internally consistent. When expressing the relative change in indicator value as a function of hydraulic roughness, it is found that wall shear stress increases, available stream power remains constant, and average flow velocity decreases (Fig. 2). The inconsistency in these trends provides practicing engineers with a dilemma.

\section{NEAR-BOUNDARY PROCESSES}

In order to explain the relevance of near-boundary processes in quantifying the relative magnitude of the erosive capacity of water it is necessary to first consider the distribution of stream power of flowing water in a water column. Such consideration provides an indication of the spatial variation of the rate of energy dissipation, which is directly related to turbulence production, and thus the relative magnitude of fluctuating pressures.

Reference [1] illustrates that the distributions of available and applied stream power differ. Available stream power is the stream power that is made available to the flowing water to overcome internal and boundary resistance. Applied stream power is the stream power that is used by the flowing water to overcome internal resistance. The latter represents the distribution of the rate of energy dissipation in the flowing water.

The difference in distribution between available and applied stream power can be explained by using an analogy of a simply supported beam bending under its own weight. The beam weight is equivalent to the available stream power, i.e. it makes a force available to bend the beam. The stresses developing within the beam re-distributes this force to overcome internal resistance offered by the beam. The distribution of the internal stresses in the beam differs from the distribution of the applied load.

Fig. 3 illustrates the distribution of available and applied stream power [1]. The available stream power has its maximum value at the water surface and is zero at the bed, while the applied stream power's maximum value is at the bed and it is zero at the water surface elevation. 
This makes sense because the greatest resistance against flow is encountered at the boundary.

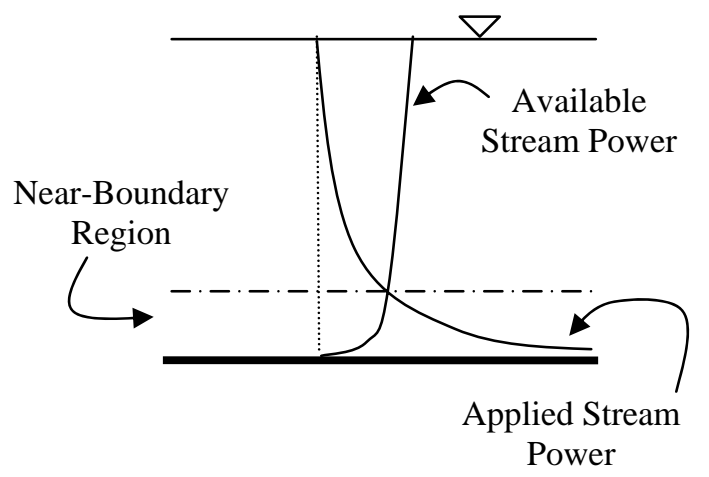

Figure 3. Distribution of available and applied stream power in flowing water.

Fig. 3 illustrates that the available stream power equals the applied stream power at the upper limit of the nearboundary region. It also shows that, theoretically, the applied stream power at the bed approaches a value of infinity. This is obviously not possible and is a limitation of the far-field equations used to derive the distribution of the applied stream power [1].

It is therefore necessary to investigate the actual distribution of the applied stream power at the boundary. Applied stream power is also known as turbulence production and its distribution within the near-boundary region is shown in Fig. 4.

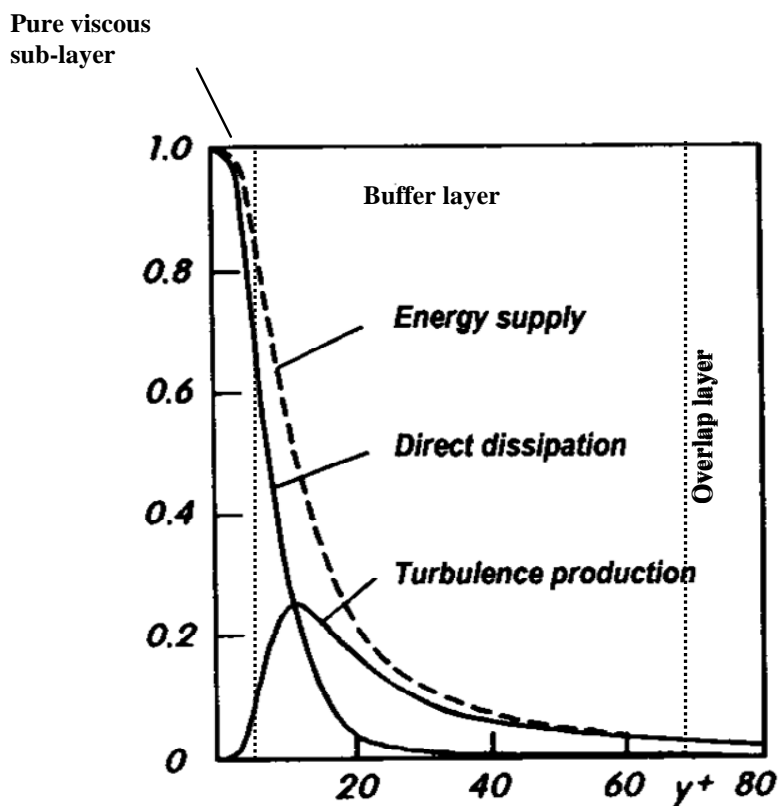

Figure 4. Dimensionless distribution of energy supply, direct dissipation and turbulence production in the near-boundary region [6]

Our principal interest for the topic under discussion is to quantify the applied stream power, and therefore the turbulence production, in the vicinity of the boundary, i.e. within the near-boundary region. Reference [5] expresses the dimensionless form of turbulence production $T P$ as,

$$
T P=\tau_{t}^{+} \cdot \frac{d u^{+}}{d y^{+}}
$$

where $\tau_{t}^{+}=\frac{\tau_{t}}{\rho u_{*}^{2}} ; \tau_{t}=$ turbulent boundary shear stress; $u_{*}=\sqrt{\frac{\bar{\tau}_{w}}{\rho}}=$ shear velocity; $\bar{\tau}_{w}=$ average wall shear stress; $u^{+}=\frac{\bar{u}}{u_{*}} ; \bar{u}=$ average velocity.

Therefore, in order to quantify the magnitude of the turbulence production in this region it is necessary to integrate the expression for dimensionless turbulence production between zero and the upper limit of this region. The near-boundary region occupies a space close to the boundary that equals about $70 y^{+}$, where $y^{+}=\delta=v / u_{*}$, and $v=$ kinematic viscosity of the water; and $\delta=$ Prandtl's wall layer thickness. Therefore, quantification of the total amount of applied stream power within this region requires integration between $y=0$ and $y=70 y^{+}$.

By fitting a curve to the dimensionless distribution of turbulence production within the near-boundary region [1] shows that,

$$
\int_{0}^{70 y^{+}} \tau_{t}^{+} \frac{d u^{+}}{d y^{+}} d y^{+}=7.853
$$

By integrating Eq. (5) it is shown that the applied stream power at the boundary, i.e. within the nearboundary region, can be expressed as,

$$
\tau_{t} \bar{u}=7.853 \cdot \rho u_{*}^{3}=7.853 \rho\left(\sqrt{\frac{\bar{\tau}_{w}}{\rho}}\right)^{3}
$$

and that the turbulent boundary shear stress can be expressed as,

$$
\tau_{t}=7.853\left(\frac{u_{*}}{\bar{u}}\right) \bar{\tau}_{w}
$$

Eq. (7) indicates that the total amount of turbulent shear stress at the boundary, resulting from turbulence production within the near-boundary region, is a function of the shear and average velocities. This equation is interpreted as representing the amount of shear stress actually applied to the boundary.

The difference between turbulent boundary shear stress and wall shear stress is similar to the difference between applied and available stream power. The wall shear stress is the amount of shear that is made available for the water to overcome internal and boundary resistance. The turbulent shear stress is the shear stress that is applied to actually overcome the internal resistance within the flow 
and at the boundary. Eq. (7) expresses the magnitude of the turbulent boundary shear stress within the nearboundary region.

When using Eq.'s (6) and (7) as indicator parameters to quantify the relative magnitude of the erosive capacity of flowing water the trends are similar. Fig. 5 shows the relationships between turbulent boundary shear stress and applied boundary stream power as a function of absolute roughness.
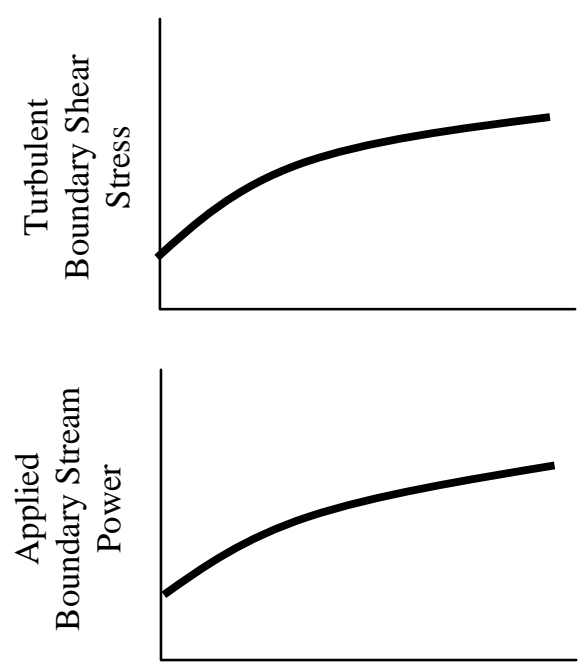

Absolute Roughness

Figure 5. Trends in turbulent boundary shear stress and applied stream power to the boundary as a function of absolute roughness [1]

\section{RELEVANCE}

The mere fact that the trends in turbulent boundary shear stress and applied stream power at the boundary are similar, as such, does not justify their use as indicator parameters. The finding that fluctuating turbulent pressure play a dominant role in incipient motion under turbulent flow conditions requires justification that these variables are related to fluctuating turbulent pressures.

Reference [6] found that the root mean square of fluctuating turbulent pressures $p^{\prime}$ is correlated to shear stress as,

$$
p^{\prime}=3 \cdot \tau_{t}
$$

While [7] found that the maximum value of fluctuating turbulent pressures $p_{\max }^{\prime}$ can be as high as $6 \cdot p^{\prime}$, which means that

$$
p_{\max }^{\prime}=18 \cdot \tau_{t}
$$

These correlations can be used as justification that a relationship between turbulent boundary shear stress and the magnitudes of fluctuating pressure exists. However, when using turbulent boundary shear stress as an indicator of the relative magnitude of the erosive capacity of turbulent flowing water it is necessary to recall that incipient motion under such conditions is not a shear process.
As applied stream power is equivalent to turbulence production and reflects the rate of energy dissipation in turbulent flowing water, it is considered to provide a good indication of the relative magnitude of fluctuating turbulent pressures. Fig. 6 shows a relationship between the rate of energy dissipation (stream power) at the base of a hydraulic jump and the standard deviation (root mean square) of fluctuating pressures.

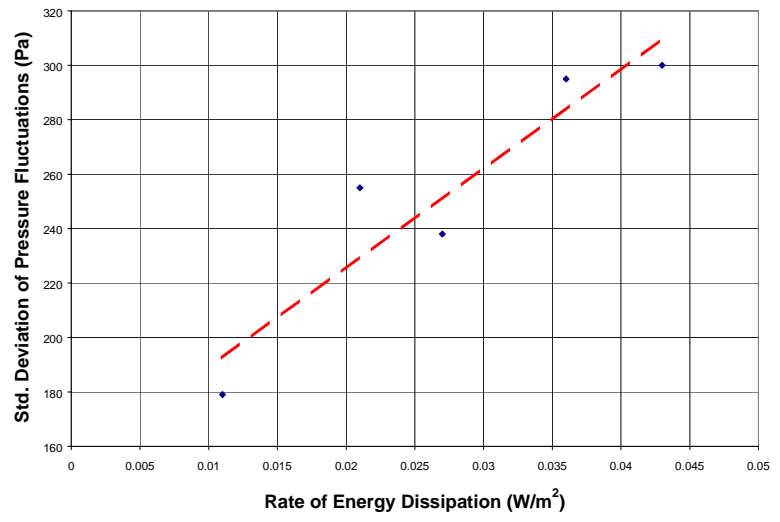

Figure 6. Relationship between stream power (rate of energy dissipation) and the standard deviation (rms) of turbulent fluctuating pressures [1]

It is therefore considered reasonable to use either turbulent boundary shear stress or applied stream power at the boundary to quantify the relative magnitude of the erosive capacity of water in turbulent flow. However, the use of stream power is considered more representative because it is a direct reflection of turbulence production and the rate of energy dissipation, and therefore the relative magnitude of turbulent pressure fluctuations.

\section{CONCLUSION}

Incipient motion under laminar flow conditions is directly dependent on shear stress, whereas incipient motion under turbulent flow conditions is principally dependent on turbulent pressure fluctuations. Laminar flow in the near-bed region requires greater effort to move a particle than is the case during turbulent flow due to the fact that shear is the principal agent leading to this motion.

When the flow conditions in the near-boundary layer is characterized as smooth turbulent flow, incipient motion is solely dependent on pressure fluctuations. If nearboundary layer flow conditions convert to rough turbulent flow, incipient motion is initiated by a combination of pressure fluctuations and horizontal flow. This results in rotation of particles during the process of incipient motion, which requires more effort to entrain particles than when smooth turbulent flow conditions exist.

When assessing incipient motion under turbulent flow conditions, the most common scenario, it is required to either quantify the actual magnitude of fluctuating pressures or use indicator parameters quantifying its relative magnitude. The actual magnitudes of fluctuating pressures are often not easily determined in practice. The preferred method for quantifying the relative magnitude of the erosive capacity of turbulent flow therefore entails the use of indicator parameters.

The preferred indicator parameters conventionally used to quantify the relative magnitude of the erosive capacity 
of water are average flow velocity, wall shear stress and available stream power. However, it has been shown that these variables are inconsistent indicators of the relative magnitude of the erosive capacity of water ([1] and [2]).

This paper presents equations that can be used to quantify the magnitude of turbulent boundary shear stress and applied boundary stream power. These parameters quantify the shear and power within the near-boundary region and are considered appropriate for quantifying the relative magnitude of the erosive capacity of water leading to incipient motion. It has also been found that the turbulent boundary shear stress and applied stream power at the boundary display consistent trends when expressed as a function of absolute hydraulic roughness.

It is concluded that turbulent boundary shear stress and applied stream power at the boundary can be used to quantify the relative magnitude of the erosive capacity of water. The use of average flow velocity to accomplish the same is not defensible, nor is the use of available stream power.

\section{REFERENCES}

[1] G.W. Annandale, Scour Technology, McGraw-Hill, New York, 2006.

[2] G.W. Annandale, Erosive Capacity, Resistance and Process, Proc. Second International Conference on Scour and Erosion, Singapore, 2004.

[3] E. Bollaert, Transient Water Pressures in Joints and Formation of Rock Scour due to High-Velocity Jet Impact, Communication No. 13. Laboratory of Hydraulic Constructions, Ecole Polytechnique Federale de Lausanne, Switzerland, 2002.

[4] R.N. Croad, Physics of Erosion of Cohesive Soils, PhD Thesis, Department of Civil Engineering, University of Auckland, Auckland, New Zealand. 1981.

[5] H. Schlicthing and K. Gersten, Boundary Layer Theory, $8^{\text {th }}$ Revised and Enlarged Edition, Springer-Verlag, New York, 2000.

[6] J.D. Hinze, Turbulence, $2^{\text {nd }}$ Edition, McGraw-Hill, New York, 1975.

[7] R. Emmerling, The instantaneous fluctuation of the wall pressure under a turbulent boundary layer flow, Max-Planck-Institut fur Stromungsforschung, Rep. No. 9, 1973. 